\title{
Dynamically generated resonances from the interaction of vector mesons with baryons
}

\author{
E. Oset* \\ Departamento de Física Teórica and IFIC, Centro Mixto Universidad de Valencia-CSIC, \\ Institutos de Investigación de Paterna, Aptdo. 22085, 46071 Valencia, Spain \\ E-mail: oset@ific.uv.es \\ P. Gonzalez, M. J. Vicente Vacas \\ Departamento de Física Teórica and IFIC, Centro Mixto Universidad de Valencia-CSIC, \\ Institutos de Investigación de Paterna, Aptdo. 22085, 46071 Valencia, Spain
}

\section{A. Ramos}

Departament d'Estructura i Constituents de la Materia, Universitat de Barcelona

\section{J. Vijande}

Departamento de Fisica Atomica Molecular y Nuclear, and IFIC, Universidad de Valencia

\section{S. Sarkar}

Variable Energy Cyclotron Centre, 1/AF, Bidhannagar, Kolkata 700064, India

\section{Bao Xi Sun}

Institute of Theoretical Physics, College of Applied Sciences, Beijing University of Technology,

Beijing 100124, China

We present the results of the first calculations involving the interaction of vector mesons with baryons, by means of which one generates a large amount of dynamically generated resonances, many of which can be associated to known resonances, while others represent predictions for new states.

International Workshop on Effective Field Theories: from the pion to the upsilon

February 2-6 2009

Valencia, Spain

\footnotetext{
*Speaker.
} 


\section{Introduction}

The combination of the information contained in the chiral Lagrangians and unitary techniques in coupled channels of mesons and baryons is an efficient tool to face many problems in Hadron Physics. In this way the interaction of the octet of pseudoscalar mesons with the octet of stable baryons has been studied and leads to $J^{P}=1 / 2^{-}$resonances which fit quite well the spectrum of the known low lying resonances with these quantum numbers $[1,2,3,4,5]$. Similarly the interaction of the octet of pseudoscalar mesons with the decuplet of baryons also leads to many resonances that can be identified with existing ones of $J^{P}=3 / 2^{-}[6,7]$. Sometimes a new resonance is predicted, as in the case of the $\Lambda(1405)$, where all the chiral approaches find two close poles rather than one, for which experimental support is presented in [8]. Another step forward in this direction has been the interpretation of low lying $J^{P}=1 / 2^{+}$states as molecular systems of two pseudoscalar mesons and one baryon $[9,10,11,12,13]$.

Much work has been done using pseudoscalar mesons as building blocks, but the consideration of vectors instead of pseudoscalars is only beginning to be exploited. In the baryon sector the interaction of the $\rho \Delta$ has been recently addressed in [14], where three degenerate $N^{*}$ states and three degenerate $\Delta$ states around $1900 \mathrm{MeV}$, with $J^{P}=1 / 2^{-}, 3 / 2^{-}, 5 / 2^{-}$, are found. The underlying theory for this study is the hidden gauge formalism $[15,16]$, which deals with the interaction of vector mesons and pseudoscalars respecting chiral dynamics, providing the interaction of pseudoscalars among themselves, with vector mesons, and vector mesons among themselves. It also offers a perspective on the chiral Lagrangians as limiting cases at low energies of vector exchange diagrams occurring in the theory. The extrapolation to $\mathrm{SU}(3)$ with the interaction of the vectors of the nonet with the baryons of the decuplet has been done in [17].

In the meson sector, the interaction of $\rho \rho$ within this formalism has been addressed in [18], where it has been shown to lead to the dynamical generation of the $f_{2}(1270)$ and $f_{0}(1370)$ meson resonances, with a branching ratio for the sensitive $\gamma \gamma$ decay channel in good agreement with experiment [19]. The extrapolation to SU(3) of the work of [18] has been done in [20], where many resonances are obtained, some of which can be associated to known meson states, while there are predictions for new ones.

In this talk we present the results of the interaction of the nonet of vector mesons with the decuplet of baryons [17] and with the octet of baryons [21], which have been done using the unitary approach in coupled channels. The scattering amplitudes lead to poles in the complex plane which can be associated to some well known resonances. Under the approximation of neglecting the three momentum of the particles versus their mass, implicit in the chiral Lagrangians, we obtain degenerate states of $J^{P}=1 / 2^{-}, 3 / 2^{-}$for the case of the interaction with the octet of baryons and $J^{P}=1 / 2^{-}, 3 / 2^{-}, 5 / 2^{-}$for the case of the interaction with the baryons of the decuplet. This degeneracy seems to be followed qualitatively by the experimental spectrum, although in some cases the spin partners have not been identified.

\section{Formalism for $V V$ interaction}

We follow the formalism of the hidden gauge interaction for vector mesons of $[15,16]$ (see also [22] for a practical set of Feynman rules). The Lagrangian involving the interaction of vector 
mesons amongst themselves is given by

$$
\mathscr{L}_{I I I}=-\frac{1}{4}\left\langle V_{\mu v} V^{\mu v}\right\rangle
$$

where the symbol \langle\rangle stands for the trace in the $S U(3)$ space and $V_{\mu v}$ is given by

$$
V_{\mu \nu}=\partial_{\mu} V_{v}-\partial_{v} V_{\mu}-i g\left[V_{\mu}, V_{v}\right]
$$

with $g$ given by $g=\frac{M_{V}}{2 f}$ with $f=93 \mathrm{MeV}$ the pion decay constant. The magnitude $V_{\mu}$ is the $S U(3)$ matrix of the vectors of the nonet of the $\rho$

$$
V_{\mu}=\left(\begin{array}{ccc}
\frac{\rho^{0}}{\sqrt{2}}+\frac{\omega}{\sqrt{2}} & \rho^{+} & K^{*+} \\
\rho^{-} & -\frac{\rho^{0}}{\sqrt{2}}+\frac{\omega}{\sqrt{2}} & K^{* 0} \\
K^{*-} & \bar{K}^{* 0} & \phi
\end{array}\right)_{\mu}
$$

The interaction of $\mathscr{L}_{I I I}$ gives rise to a contact term coming from $\left[V_{\mu}, V_{v}\right]\left[V_{\mu}, V_{v}\right]$

$$
\mathscr{L}_{I I I}^{(c)}=\frac{g^{2}}{2}\left\langle V_{\mu} V_{v} V^{\mu} V^{v}-V_{v} V_{\mu} V^{\mu} V^{v}\right\rangle
$$

and on the other hand it gives rise to a three vector vertex from

$$
\mathscr{L}_{I I I}^{(3 V)}=i g\left\langle\left(\partial_{\mu} V_{v}-\partial_{v} V_{\mu}\right) V^{\mu} V^{v}\right\rangle=i g\left\langle\left(V^{\mu} \partial_{v} V_{\mu}-\partial_{v} V_{\mu} V^{\mu}\right) V^{v}\right\rangle,
$$

In this latter case one finds an analogy with the coupling of vectors to pseudoscalars given in the same theory by

$$
\mathscr{L}_{V P P}=-i g \operatorname{tr}\left(\left[P, \partial_{\mu} P\right] V^{\mu}\right),
$$

where $P$ is the $\mathrm{SU}(3)$ matrix of the pseudoscalar fields.

In a similar way, we have the Lagrangian for the coupling of vector mesons to the baryon octet given by $[28,29]^{1}$

$$
\mathscr{L}_{B B V}=\frac{g}{2}\left(\operatorname{tr}\left(\bar{B} \gamma_{\mu}\left[V^{\mu}, B\right]+\operatorname{tr}\left(\bar{B} \gamma_{\mu} B\right) \operatorname{tr}\left(V^{\mu}\right)\right),\right.
$$

where $B$ is now the SU(3) matrix of the baryon octet [23]. Similarly, one has also a lagrangian for the coupling of the vector mesons to the baryons of the decuplet, which can be found in [25].

With these ingredients we can construct the Feynman diagrams that lead to the $P B \rightarrow P B$ and $V B \rightarrow V B$ interaction, by exchanging a vector meson between the pseudoscalar or the vector meson and the baryon, as depicted in Fig.1 .

By looking at the Lagrangian of eq. (2.5) we see that the field $V^{v}$ cannot correspond to an external vector meson. Indeed, if this were the case, the $v$ index must be spatial, because $\varepsilon^{0}$ is zero in the limit of zero three momentum, and then the partial derivative $\partial_{v}$ leads to a three momentum of the vector mesons which are neglected in the approach. Then $V^{v}$ corresponds to the exchanged vector and the analogy with the pseudoscalar and vector interaction is much closer (see Eq. (2.6).

\footnotetext{
${ }^{1}$ Correcting a misprint in [28]
} 
[a]
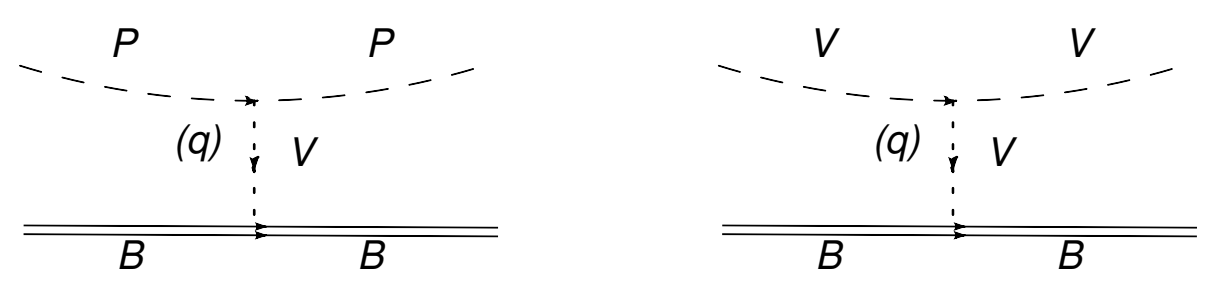

Figure 1: Diagrams obtained in the effective chiral Lagrangians for interaction of pseudoscalar [a] or vector [b] mesons with the octet or decuplet of baryons.

Actually, they are formally identical substituting the octet of pseudoscalar fields by the octet of the vector fields, with the additional factor $\vec{\varepsilon} \vec{\varepsilon}^{\prime}$ in the case of the interaction of the vector mesons. Note that $\varepsilon_{\mu} \varepsilon^{\mu}$ in Eq. (2.5) becomes $-\vec{\varepsilon} \vec{\varepsilon}^{\prime}$ and the signs of the Lagrangians also agree.

A small amendment is in order in the case of vector mesons, which is due to the mixing of $\omega_{8}$ and the singlet of $\mathrm{SU}(3), \omega_{1}$, to give the physical states of the $\omega$ and the $\phi$. In this case, all one must do is to take the matrix elements known for the $P B$ interaction and wherever $P$ is the $\eta_{8}$ multiply the amplitude by the factor $1 / \sqrt{3}$ to get the corresponding $\omega$ contribution and by $-\sqrt{2 / 3}$ to get the corresponding $\phi$ contribution. Upon the approximation consistent with the neglect of the three momentum versus the mass of the particles (in this case the baryon), we can just take the $\gamma^{0}$ component of eq. (2.7) and then the transition potential corresponding to the diagram of fig 1(b ) is given by

$$
V_{i j}=-C_{i j} \frac{1}{4 f^{2}}\left(k^{0}+k^{\prime 0}\right) \vec{\varepsilon} \vec{\varepsilon}^{\prime},
$$

where $k^{0}, k^{\prime 0}$ are the energies of the incoming and outgoing vector mesons. The same occurs in the case of the decuplet.

The $C_{i, j}$ coefficients of eq. (2.8) can be obtained directly from [2, 26, 27] with the simple rules given above for the $\omega$ and the $\phi$, and substituting $\pi$ by $\rho$ and $K$ by $K^{*}$ in the matrix elements. The coefficients are obtained both in the physical basis of states or in the isospin basis. Here we will show results in isospin basis. The coefficients for the case of the decuplet can be found in [7].

The next step to construct the scattering matrix is done by solving the coupled channels Bethe Salpeter equation in the on shell factorization approach of $[2,3]$

$$
T=[1-V G]^{-1} V
$$

with $\mathrm{G}$ the loop function of a vector meson and a baryon which we calculate in dimensional regularization using the formula of [3] and similar values for the subtraction constants. The G function is convoluted with the spectral function for the vector mesons to take into account their width.

The iteration of diagrams implicit in the Bethe Salpeter equation in the case of the vector mesons propagates the $\vec{\varepsilon} \vec{\varepsilon}^{\prime}$ term of the interaction, thus, the factor $\vec{\varepsilon} \vec{\varepsilon}^{\prime}$ appearing in the potential V, factorizes also in the $\mathrm{T}$ matrix for the external vector mesons. 


\section{Results}

In this section we show results for the amplitudes obtained in the attractive channels mentioned above. Since the spin dependence only comes from the $\vec{\varepsilon} \vec{\varepsilon}^{\prime}$ factor and there is no dependence on the spin of the baryons, the interaction for vector-baryon states with $1 / 2^{-}$and $3 / 2^{-}$is the same and then we get two degenerate states each time with the two spins. In the case of the decuplet we get degeneracy for $1 / 2^{-}, 3 / 2^{-}, 5 / 2^{-}$.

We summarize the results in the two Tables below

\begin{tabular}{|c|c|c|c|c|c|c|c|c|}
\hline \multirow[t]{3}{*}{$S, I$} & \multicolumn{3}{|c|}{ Theory } & \multicolumn{5}{|c|}{ PDG data } \\
\hline & \multirow[t]{2}{*}{$\begin{array}{l}\text { pole position } \\
\text { (convolution) }\end{array}$} & \multicolumn{2}{|c|}{$\begin{array}{c}\text { real axis } \\
\text { (convolution) }\end{array}$} & \multirow[b]{2}{*}{ name } & \multirow[b]{2}{*}{$J^{P}$} & \multirow[b]{2}{*}{ status } & \multirow[b]{2}{*}{ mass } & \multirow[b]{2}{*}{ width } \\
\hline & & mass & width & & & & & \\
\hline \multirow[t]{4}{*}{$0,1 / 2$} & - & 1696 & 92 & $N(1650)$ & $1 / 2^{-}$ & $\star \star \star \star \star$ & $1645-1670$ & $145-185$ \\
\hline & & & & $N(1700)$ & $3 / 2^{-}$ & $\star \star \star$ & $1650-1750$ & $50-150$ \\
\hline & $1977+\mathrm{i} 53$ & 1972 & 64 & $N(2080)$ & $3 / 2^{-}$ & $\star \star$ & $\approx 2080$ & $180-450$ \\
\hline & & & & $N(2090)$ & $1 / 2^{-}$ & $\star$ & $\approx 2090$ & $100-400$ \\
\hline \multirow[t]{4}{*}{$-1,0$} & $1784+\mathrm{i} 4$ & 1783 & 9 & $\Lambda(1690)$ & $3 / 2^{-}$ & $\star \star \star \star \star$ & $1685-1695$ & $50-70$ \\
\hline & & & & $\Lambda(1800)$ & $1 / 2^{-}$ & $\star \star \star$ & $1720-1850$ & $200-400$ \\
\hline & $1907+\mathrm{i} 70$ & 1900 & 54 & $\Lambda(2000)$ & $?$ & $\star$ & $\approx 2000$ & $73-240$ \\
\hline & $2158+\mathrm{i} 13$ & 2158 & 23 & & & & & \\
\hline \multirow[t]{3}{*}{$-1,1$} & --- & 1830 & 42 & $\Sigma(1750)$ & $1 / 2^{-}$ & $\star \star \star$ & $1730-1800$ & $60-160$ \\
\hline & --- & 1987 & 240 & $\Sigma(1940)$ & $3 / 2^{-}$ & $\star \star \star$ & $1900-1950$ & $150-300$ \\
\hline & & & & $\Sigma(2000)$ & $1 / 2^{-}$ & $\star$ & $\approx 2000$ & $100-450$ \\
\hline \multirow[t]{2}{*}{$-2,1 / 2$} & $2039+\mathrm{i} 67$ & 2039 & 64 & $\Xi(1950)$ & $?$ & $\star \star \star$ & $1950 \pm 15$ & $60 \pm 20$ \\
\hline & $2083+\mathrm{i} 31$ & 2077 & 29 & $\Xi(2120)$ & $?$ & $\star$ & $\approx 2120$ & 25 \\
\hline
\end{tabular}

Table 1: The properties of the 9 dynamically generated resonances and their possible PDG counterparts.

As one can see in Table 1 there are states which one can easily associate to known resonances. There are ambiguities in other cases. One can also see that in several cases the degeneracy in spin that the theory predicts is clearly visible in the experimental data, meaning that there are several states with about $50 \mathrm{MeV}$ or less mass difference between them. In some cases, the theory predicts quantum numbers for resonances which have no spin and parity associated. It would be interesting to pursue the experimental research to test the theoretical predictions. 


\begin{tabular}{|c|c|c|c|c|c|c|c|c|}
\hline \multirow[t]{3}{*}{$S, I$} & \multicolumn{3}{|c|}{ Theory } & \multicolumn{5}{|c|}{ PDG data } \\
\hline & \multirow[t]{2}{*}{ pole position } & \multicolumn{2}{|l|}{ real axis } & \multirow[t]{2}{*}{ name } & \multirow[t]{2}{*}{$J^{P}$} & \multirow[t]{2}{*}{ status } & \multirow[t]{2}{*}{ mass } & \multirow[t]{2}{*}{ width } \\
\hline & & mass & width & & & & & \\
\hline \multirow[t]{3}{*}{$0,1 / 2$} & \multirow[t]{3}{*}{$1850+i 5$} & \multirow[t]{2}{*}{1850} & \multirow[t]{3}{*}{11} & $N(2090)$ & $1 / 2^{-}$ & $\star$ & $1880-2180$ & $95-414$ \\
\hline & & & & $N(2080)$ & $3 / 2^{-}$ & $\star \star$ & 1804-2081 & $180-450$ \\
\hline & & $2270($ bump $)$ & & $N(2200)$ & $5 / 2^{-}$ & $\star \star$ & $1900-2228$ & $130-400$ \\
\hline \multirow[t]{4}{*}{$0,3 / 2$} & \multirow[t]{4}{*}{$1972+i 49$} & \multirow[t]{3}{*}{1971} & \multirow[t]{4}{*}{52} & $\Delta(1900)$ & $1 / 2^{-}$ & $\star \star$ & $1850-1950$ & $140-240$ \\
\hline & & & & $\Delta(1940)$ & $3 / 2^{-}$ & $\star$ & $1940-2057$ & $198-460$ \\
\hline & & & & $\Delta(1930)$ & $5 / 2^{-}$ & $\star \star \star$ & $1900-2020$ & $220-500$ \\
\hline & & $2200($ bump $)$ & & $\Delta(2150)$ & $1 / 2^{-}$ & $\star$ & $2050-2200$ & $120-200$ \\
\hline$-1,0$ & $2052+i 10$ & 2050 & 19 & $\Lambda(2000)$ & $?$ & $\star$ & $1935-2030$ & $73-180$ \\
\hline \multirow[t]{4}{*}{$-1,1$} & $1987+i 1$ & 1985 & 10 & $\Sigma(1940)$ & $3 / 2^{-}$ & $\star \star \star$ & $1900-1950$ & $150-300$ \\
\hline & $2145+i 58$ & 2144 & 57 & $\Sigma(2000)$ & $1 / 2^{-}$ & $\star$ & $1944-2004$ & $116-413$ \\
\hline & \multirow[t]{2}{*}{$2383+i 73$} & \multirow[t]{2}{*}{2370} & \multirow[t]{2}{*}{99} & $\Sigma(2250)$ & $?$ & $\star \star \star$ & $2210-2280$ & $60-150$ \\
\hline & & & & $\Sigma(2455)$ & $?$ & $\star \star$ & $2455 \pm 10$ & $100-140$ \\
\hline \multirow[t]{3}{*}{$-2,1 / 2$} & $2214+i 4$ & 2215 & 9 & $\Xi(2250)$ & $?$ & $\star \star$ & $2189-2295$ & $30-130$ \\
\hline & $2305+i 66$ & 2308 & 66 & $\Xi(2370)$ & $?$ & $\star \star$ & $2356-2392$ & $75-80$ \\
\hline & $2522+i 38$ & 2512 & 60 & $\Xi(2500)$ & $? ?$ & $\star$ & $2430-2505$ & $59-150$ \\
\hline$-3,1$ & $2449+i 7$ & 2445 & 13 & $\Omega(2470)$ & $?$ & $\star \star$ & $2474 \pm 12$ & $72 \pm 33$ \\
\hline
\end{tabular}

Table 2: The properties of the 10 dynamically generated resonances and their possible PDG counterparts. We also include the $N^{*}$ bump around $2270 \mathrm{MeV}$ and the $\Delta^{*}$ bump around $2200 \mathrm{MeV}$.

The results for the decuplet, summarized in Table 2, are equally interesting. We observe here that one gets $\Delta$ states as well as $N^{*}$, some of which can be clearly identified with known resonances. It is also nice to see the spin degeneracy present in $N^{*}, \Delta$ and $\Sigma$ experimental data. The predictions made here for resonances not observed should be a stimulus for further search of such states. In this sense it is worth noting the experimental program at Jefferson Lab [30] to investigate the $\Xi$ resonances. We are confident that the predictions shown here stand on solid grounds and anticipate much progress in the area of baryon spectroscopy and on the understanding of the nature of the baryonic resonances. 


\section{Acknowledgments}

This work is partly supported by DGICYT contract number FIS2006-03438. This research is part of the EU Integrated Infrastructure Initiative Hadron Physics Project under contract number RII3-CT-2004-506078.

\section{References}

[1] N. Kaiser, P. B. Siegel and W. Weise, Phys. Lett. B 362, 23 (1995).

[2] E. Oset and A. Ramos, Nucl. Phys. A 635 (1998) 99 .

[3] J. A. Oller and U. G. Meissner, Phys. Lett. B 500, 263 (2001).

[4] C. Garcia-Recio, J. Nieves and L. L. Salcedo, Phys. Rev. D 74 (2006) 034025.

[5] T. Hyodo, S. I. Nam, D. Jido and A. Hosaka, Phys. Rev. C 68, 018201 (2003).

[6] E. E. Kolomeitsev and M. F. M. Lutz, Phys. Lett. B 585 (2004) 243.

[7] S. Sarkar, E. Oset and M. J. Vicente Vacas, Nucl. Phys. A 750 (2005) 294 [Erratum-ibid. A 780 (2006) 78] .

[8] V. K. Magas, E. Oset and A. Ramos, Phys. Rev. Lett. 95, 052301 (2005).

[9] A. Martinez Torres, K. P. Khemchandani and E. Oset, Phys. Rev. C 77, 042203 (2008).

[10] A. Martinez Torres, K. P. Khemchandani and E. Oset, Eur. Phys. J. A 35, 295 (2008) .

[11] K. P. Khemchandani, A. Martinez Torres and E. Oset, Eur. Phys. J. A 37, 233 (2008) .

[12] D. Jido and Y. Kanada-En'yo, Phys. Rev. C 78, 035203 (2008).

[13] Y. Kanada-En'yo and D. Jido, Phys. Rev. C 78, 025212 (2008).

[14] P. Gonzalez, E. Oset and J. Vijande, Phys. Rev. C79, 025209 (2009)

[15] M. Bando, T. Kugo, S. Uehara, K. Yamawaki and T. Yanagida, Phys. Rev. Lett. 54, 1215 (1985).

[16] M. Bando, T. Kugo and K. Yamawaki, Phys. Rept. 164, 217 (1988).

[17] S. Sarkar, B. X. Sun, E. Oset and M. J. V. Vacas, arXiv:0902.3150 [hep-ph].

[18] R. Molina, D. Nicmorus and E. Oset, Phys. Rev. D 78, 114018 (2008) .

[19] H. Nagahiro, J. Yamagata-Sekihara, E. Oset and S. Hirenzaki, arXiv:0809.3717 [hep-ph].

[20] L. S. Geng and E. Oset, Phys. Rev. D79 (2009) 074009 arXiv:0812.1199 [hep-ph].

[21] A. Ramos and E. Oset, to be published.

[22] H. Nagahiro, L. Roca, A. Hosaka and E. Oset, Phys. Rev. D 79, 014015 (2009)

[23] G. Ecker, Prog. Part. Nucl. Phys. 35 (1995) 1

[24] V. Bernard, N. Kaiser and U. G. Meissner, Int. J. Mod. Phys. E4 (1995) 193

[25] E. E. Jenkins and A. V. Manohar, Phys. Lett. B 259, 353 (1991).

[26] A. Ramos, E. Oset and C. Bennhold, Phys. Rev. Lett. 89, 252001 (2002).

[27] T. Inoue, E. Oset and M. J. Vicente Vacas, Phys. Rev. C 65, 035204 (2002).

[28] F. Klingl, N. Kaiser and W. Weise, Nucl. Phys. A 624 (1997) 527.

[29] J. E. Palomar and E. Oset, Nucl. Phys. A 716, 169 (2003).

[30] J. W. Price et al. [CLAS Collaboration], Phys. Rev. C 71, 058201 (2005). 